\title{
The Role of Serum Neuron Specific Enolase in Detecting Preoperative Brain Injury in Infants and Toddlers with Congenital Heart Disease
}

\author{
AbdelGhaffar HM (1), ElAwady HA (2), Eid MMA (3) and Abozeid RA (4). \\ (1) Professor of Pediatrics, Pediatric department, Faculty of Medicine Fayoum University, \\ Fayoum, Egypt. \\ (2) Lecturer of Pediatrics, Pediatric department, Faculty of Medicine Fayoum university, \\ Fayoum, Egypt. \\ (3) Lecturer of Clinical Pathology, Clinical Pathology department, Faculty of Medicine \\ Fayoum University, Fayoum, Egypt. \\ (4) Department of Pediatrics, National Heart Institute, Cairo, Egypt.
}

Corresponding author: Radwa AbdulHarris Abozeid, Pediatric department, National Heart Institute, Cairo, Egypt

E-mail address: r.abozeid@gmail.com

Tel: +201008031179

\section{ABSTRACT}

A significant number of patients presenting with Congenital heart diseases (CHD) experience concomitant neurological disorders, whether subclinical or overt. These neurological manifestations of brain injury may become manifest after undergoing surgery for CHD correction. Therefore, risk stratifying patients with high chances of developing adverse neurological outcomes before undergoing their surgeries can decrease these unfavorable outcomes by ensuring optimal surgical precautions. Serum neuron-specific enolase (SNSE), a known marker of neuronal damage, has been used in this study to detect level of brain injury. The purpose of this study is to investigate the role of serum neuron-specific enolase in detecting brain injury in infants and toddlers with congenital heart disease prior to performing surgery. This study seeks to answer the research question, can SNSE demonstrate the presence of a significant incidence of baseline brain damage in CHD patients, before undergoing any interventions.

KEYWORDS: Congenital heart disease, neuronal damage, serum neuron-specific enolase, perioperative brain injury.

\section{INTRODUCTION}

Cerebral damage is a serious complication of pediatric cardiac surgery in infants with congenital heart disease. Identifying infants at risk of brain injury preoperatively can be useful in counseling of parents and in early diagnosis and rehabilitation therapy and would aid in developing therapeutic strategies to limit perioperative cerebral damage [1].

The presence of preexisting neurological problems preoperatively share in increasing the risk of adverse neurological outcomes after surgery [2]. 
The use of serum brain markers for diagnosis of neuronal injury have long been implicated in early detection of brain injury, Neuronspecific enolase has been advocated in many studies of such kind, an example is its use for detection of hypoxic ischemic encephalopathy in cases of perinatal asphyxia for the application of neuroprotective strategies[3].

While previous researchers have focused on the postoperative period in their investigation of brain vulnerability, the pattern of preoperative background of neurological status has not been well reported in this population of children suffering from congenital heart disease. It is believed that the presence of comorbidity and vulnerability of these infants makes this an important timeframe to study [4].

The aim of this study was to use serum neuron-specific enolase (sNSE) to detect the level of brain injury in infants and toddlers with congenital heart disease prior to performing corrective heart surgery.

\section{Methods}

All relevant studies were identified by computerized searches of PubMed using the following Medical Subject Headings and keywords term: biomarker, cardiac surgery, brain injury and neurological complication.

\section{Epidemiology of congenital heart disease}

Congenital heart disease (CHD) is the most common cause of major congenital anomalies, representing a major global health problem. Twenty-eight percent of all major congenital anomalies consist of heart defects [5]. Congenital malformations of the heart are anomalies formed during embryogenesis that evolve during gestation, survive during intrauterine life, and present during the course of extra-uterine life. [6]. The incidence of CHD in different studies varies from about 4/1,000 to 50/1,000 live births. [7].

Over time, the reported total CHD birth prevalence increased substantially (Fig. 1A) from 0.6 per 1,000 live births in 1930 to 1934 to 9.1 per 1,000 live births after 1995. This corresponds to 1.35 million newborns with CHD every year, representing a major global health burden [8].

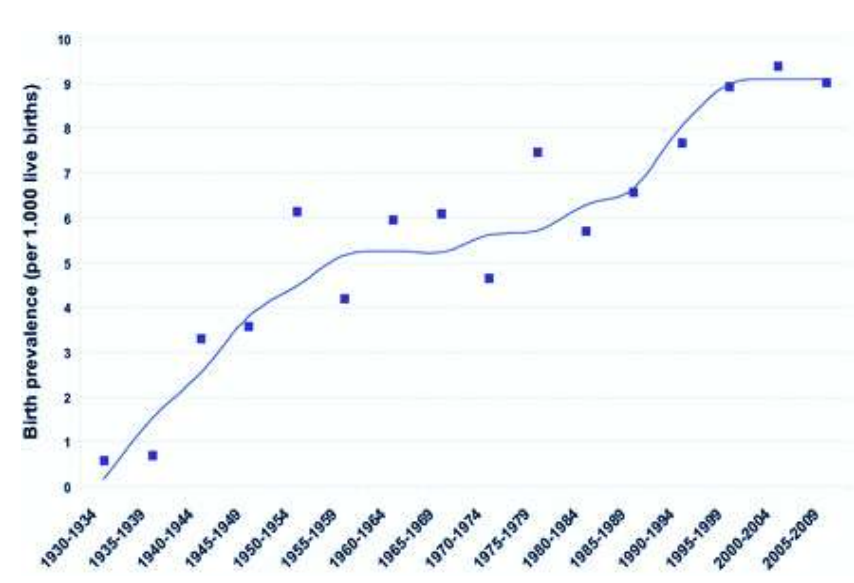

Fig. 1A. Total CHD birth prevalence over time. Time course of reported total congenital heart disease (CHD) birth prevalence from 1930 untill2010. The blue line shows the time trend and the squares represent the calculated birth prevalence values for each time period (van der Linde et al., 2011).

Neurological affection in children with congenital heart disease

Previously, studies of neurological outcomes in children with CHD have focused on factors 
related to surgery, when cerebral perfusion may be compromised during cardiopulmonary bypass, deep hypothermic circulatory arrest, general anesthesia, etc. However, the fact that these infants are at risk for adverse outcome before entering the operating room is supported by an increasing body of literature. Brain abnormalities are detectable by preoperative neuroimaging and neurological examination in a significant percentage of infants with CHD. These findings are multifactorial, such as concomitant associated factors effecting both systems causing both congenital brain abnormalities and cardiac anomalies, genetic causes, combined cardiac and brain manifestations of inherited metabolic disease or developmental factors contributed to by intrauterine hemodynamic alterations and acquired brain injury related to prolonged cyanosis or hypoperfusion after birth [9].

Anthropometric data collected from a regional case control study revealed that infants with CHD had abnormal in utero somatic growth compared to matched controls [10].

Chromosomal abnormality has been made responsible for multiple associations of $\mathrm{CHD}$ and CNS anomalies. Some authors classified them into syndromic lesions where chromosomal evidence of association was established and non-syndromic where genetic evidence was not confirmed as the cause of association [11].

Hence, it can be strongly stated that neurological derangements in children with congenital heart disease are present even before undergoing surgery, such derangements might be subtle or clinically covert and surgery only attributes in amplifying them, thus surgery is not the cause but instead a complicating factor.

\section{Biochemical markers used to detect brain injury}

The majority of studies that have previously assessed potential biomarkers for neurologic injury in children with CHD undergoing heart surgery have focused on neuron-specific enolase (NSE) and S-100 $\beta$ [12]. Alpha IIspectrin breakdown products has also been advocated in recent studies and were found to be detected in the serum of neonates with congenital heart disease in the perioperative period and levels increased to a greater degree in infants following open-heart surgery [13]. Glial fibrillary acidic protein, tau protein, matrix metallopro-teinase-9, ubiquitin $\mathrm{C}$ terminal hydroxylase-L1 and neurofilaments were employed in same interest [14].

These biomarkers, independently of clinical and radiological findings, give a general picture of cerebral situation at the cellular level and the degree of brain dysfunction.

\section{Serum Neuron specific enolase}

NSE is an isoenzyme of enolase (2-phosphoD-glycerate hydrolase), which catalyzes the transition of 2-phosphoglycerate into phosphoenolpyruvate, the ninth step of glycolysis [15].

NSE was engaged in many researches in monitoring the degree of brain damage in traumatic brain injury [16]. NSE also played an important role in the prediction of the outcome of patients who had brain ischemia or bleeding or after they underwent a cardiac surgery or a neurosurgery [17]. NSE is also produced by some tumor cells of neuro- 
ectodermal origin. Furthermore, NSE along with S100B protein (a structural protein used as a marker of brain injury), were researched in relation to prion diseases of CNS [18].

Although NSE is fairly specific for brain tissue, has low variability with regard to sex and age, and is rapidly detected in serum following injury, in several studies, it was unable to differentiate mild injury from absence of injury and has been shown to be released during hemolysis [13].

\section{Serum NSE as a biomarker in risk stratifying patients with CHD before surgery}

NSE is a human $\gamma$ enolase and is one of the biomarkers used to detect neuronal tissue damage [19].

Serum NSE levels correlated with the extent of brain damage and in some studies with prognosis, particularly in neonates after hypoxic ischemic brain injury [20].

Literature by Trakas et al., [21] who conducted a prospective observational study including neonates with congenital heart disease undergoing cardiopulmonary bypass surgery, where NSE and s100B were measured from serum samples obtained preoperatively, immediately postoperatively, and once daily on postoperative days 1-7, concluded that the drop in sNSE levels to below preoperative level was due to preexisting prenatal brain injury that could be enhanced by longer surgical times.

The findings are clearly contradicted by those of Simsic et al., [22] whose study tested whether brain injury may be detected with a blood test for specific biomarkers in neonates undergoing cardiopulmonary bypass. They found that preoperative NSE levels were not predictors of abnormal preoperative brain MRI. However, they stated the level of evidence to be $(\mathrm{p}=0.4)$.

According to Trakas et al., [21] the negative correlation between NSE levels and brain structural abnormalities scores (which contradicted the anticipated opposite result) is since some neonates with complex congenital heart disease have a decreased neuronal volume at birth and therefore less volume of neurons capable of cell death leading to generally lower NSE levels (when compared with children without CHD with normal neuronal tissue mass).

Another study performed in order to find the potential role of systemic inflammation on brain injury in newborns with congenital heart disease (CHD) by measuring levels of central nervous system (CNS)-derived proteins (phosphorylated neurofilament-heavy subunit (pNF-H), neuron-specific enolase (NSE) and S100B in serum prior to and following cardiac surgery [23], stated that the amount of NSE in patient sera prior to surgery did not differ significantly from that in normal cord blood.

Schmitt et al., [24], investigated whether neuron-specific enolase (NSE) in serum or cerebrospinal fluid (CSF) reflects subtle or manifest brain injury in children undergoing cardiac surgery using cardiopulmonary bypass (CPB) and concluded that the value of s-NSE to ascertain brain injury in children undergoing cardiac surgery with $\mathrm{CPB}$ is highly questionable.

A study performed by Hoffmann et al., (2017)[7], on adult population to investigate 
the association between serum NSE levels, body mass index (BMI), total gray matter volume (GMV), and magnetic resonance imaging-based indices of aging, hypothesized that increased serum NSE levels are a result of obesity-associated structural damage of gray matter observed in BMI $<25$.

\section{DISCUSSION}

Neurological complications contribute significantly to mortality and morbidity from congenital heart disease with serious longterm consequences. Congenital heart disease has been shown to adversely influence the neuro-developmental outcome of children in many ways [25]. Recent advances in medical management, trans-cutaneous catheter intervention, surgical procedures, and cardiopulmonary bypass, have significantly increased the survival rate of such children. However, up to $50 \%$ of children with congenital heart disease (CHD) experience neurodevelopmental and behavioral problems including seizures, cognitive impairment, delays in speech, language, visual-motor and visual-spatial skills, attention deficit/hyperactivity disorder and learning disabilities[26].

It has for long being debated, wither the resultant neurological complications arising in children after surgery were due to the impact of surgery alone or due to predisposition to neurological affection due to Congenital heart disease in first place [23]. The developing fetus with congenital heart defects may be exposed to hypoxia and abnormal cerebral perfusion in utero and these insults on the brain may continue during the neonatal period because of the failing heart [27].
Up to date, there are promising data on the potential use of biomarkers, but there is no marker fully suitable for the detection of NC after CS, which shows the mechanisms leading to the development of these neurological injuries [28].

The biomarkers that appeared to be better indicators of brain injury severity before and after cardiac surgery were are S100B protein, neuron-specific enolase, glial fibrillary acidic protein, tau protein, matrix metalloproteinase9, ubiquitin $\mathrm{C}$ terminal hydroxylase-L1 and neurofilaments [28].

It is fair to mention that the use of SNSE to detect brain injury might be subjected to limitations, an important fact is the nature of NSE, the non-specificity of the test may be due to the presence of NSE in platelets and erythrocytes [29] and hence, even limited hemolysis could substantially increase NSE levels in plasma. NSE is also located in peripheral neuroendocrine cells, which has encouraged studies evaluating its use as a marker of neuroendocrine tumors such as non-small cell lung carcinoma. However, the implementation of spectrometry to exclude cases of covert hemolysis is a respectable solution.

During CS, various intra-operative factors significantly influence the concentration of NSE. The use of both extracorporeal pump and cardiotomy suction has been associated with increased levels of NSE, although, in the case of the latest one, it is possible that these levels arise because of contamination from sources of non-neuronal enolase [30].

In the study by Liu et al. [12]conducted with 40 children who had congenital heart disease, the 20 children that underwent CS presented 
higher levels of NSE compared to those who underwent other types of interventional therapy, this shows that the type of intervention may have an impact on results when using sNSE to detect brain injury.

Further studies are to be conducted with large number of patients having different CHD types to find if any relation does exist in relation to age, sex or BMI and whether level of SNSE differs according to the type of congenital heart anomaly.

Careful preoperative assessment of $\mathrm{CHD}$ patients, especially cyanotic CHD, before surgery or interventional treatment, in order to risk stratify patients and pick out those at high risk of adverse outcomes of CHD surgery and intervention so brain protective measures could be taken.

It is also fair to mention that in this literature, comparisons between studies were difficult because various brain markers and methods were used to assess brain injury in infants with complex CHD.

\section{CONCLUSION}

In conclusion, serum NSE levels were found to be significantly higher in patients with CHD compared to control group. This proves evidence of pre-existing brain injury among those subjects, even before undergoing surgery. Physicians and other caregivers should be more aware of the vulnerability of the brain and of the possible hypoxia and/or ischemia in infants with CHD, that may lead to adverse outcomes after corrective surgery. This study is thus in favor of the assumption that biochemical monitoring and risk stratification of children with CHD at risk of adverse neurological outcomes after surgery is possible. Further investigations are needed to strengthen the hypothesis and include serum NSE in routine preoperative investigations of patients before surgery. 


\section{REFERENCES}

[1] S. Talwar et al., "Neurological injury in paediatric cardiac surgery," Indian J. Thorac. Cardiovasc. Surg., vol. 33, no. 1, pp. 15-28, 2017.

[2] N. S. Abend, D. J. Dlugos, and R. R. Clancy, "A review of long-term EEG monitoring in critically ill children with hypoxic-ischemic encephalopathy, congenital heart disease, ECMO, and stroke," J. Clin. Neurophysiol. Off. Publ. Am. Electroencephalogr. Soc., vol. 30, no. 2, p. 134, 2013.

[3] D. Kelen, C. Andorka, M. Szabó, A. Alafuzoff, K. Kaila, and M. Summanen, "Serum copeptin and neuron specific enolase are markers of neonatal distress and long-term neurodevelopmental outcome," PLoS One, vol. 12, no. 9, p. e0184593, 2017.

[4] B. Mehta, R. Hunt, K. Walker, and N. Badawi, "Evaluation of preoperative amplitude-integrated electroencephalography (aEEG) monitoring for predicting long-term neurodevelopmental outcome among infants undergoing major surgery in the neonatal period," J. Child Neurol., vol. 31, no. 11, pp. 1276-1281, 2016.

[5] H. Dolk, M. Loane, E. Garne, and a E. S. of C. A. (EUROCAT) W. Group, "Congenital heart defects in Europe: prevalence and perinatal mortality, 2000 to 2005," Circulation, vol. 123, no. 8, pp. 841-849, 2011.

[6] J. K. Perloff and A. Marelli, Perloff's Clinical Recognition of Congenital Heart Disease: Expert Consult-Online and Print. Elsevier Health Sciences, 2012.

[7] J. Hoffmann et al., "Association between serum neuron-specific enolase, age, overweight, and structural MRI patterns in 901 subjects," Transl. Psychiatry, vol. 7, no. 12, pp. 1-9, 2017.

[8] D. Van Der Linde et al., "Birth prevalence of congenital heart disease worldwide: a systematic review and meta-analysis," J. Am. Coll. Cardiol., vol. 58, no. 21, pp. 2241-2247, 2011.

[9] M. T. Donofrio and A. N. Massaro, "Impact of congenital heart disease on brain development and neurodevelopmental outcome," Int. J. Pediatr., vol. 2010, 2010.

[10] G. L. Rosenthal, "Patterns of prenatal growth among infants with cardiovascular malformations: possible fetal hemodynamic effects," Am. J. Epidemiol., vol. 143, no. 5, pp. 505513, 1996.

[11] A. Egbe, S. Lee, D. Ho, S. Uppu, and S. Srivastava, "Prevalence of congenital anomalies in newborns with congenital heart disease diagnosis," Ann. Pediatr. Cardiol., vol. 7, no. 2, p. 86, 2014.

[12] Y. Liu, Y. Xu, D. Li, Y. Shi, and M. Ye, "Comparison of S100B and NSE between cardiac surgery and interventional therapy for children," Pediatr. Cardiol., vol. 30, no. 7, pp. 893-897, 2009.

[13] P. Jain, M. C. Spaeder, M. T. Donofrio, P. Sinha, R. A. Jonas, and R. J. Levy, "Detection of alpha ii-spectrin breakdown products in the serum of neonates with congenital heart disease," Pediatr. Crit. care Med. a J. Soc. Crit. Care Med. World Fed. Pediatr. Intensive Crit. Care Soc., vol. 
15, no. 3, p. 229, 2014.

[14] C. Hernández-García, A. RodríguezRodríguez, and J. J. Egea-Guerrero, "Brain injury biomarkers in the setting of cardiac surgery: still a world to explore," Brain Inj., vol. 30, no. 1, pp. 10-17, 2016.

[15] L. Ramont, H. Thoannes, A. Volondat, F. Chastang, M.-C. Millet, and F.-X. Maquart, "Effects of hemolysis and storage condition on neuron-specific enolase (NSE) in cerebrospinal fluid and serum: implications in clinical practice," Clin. Chem. Lab. Med., vol. 43, no. 11, pp. 1215-1217, 2005.

[16] H. Zetterberg, D. H. Smith, and K. Blennow, "Biomarkers of mild traumatic brain injury in cerebrospinal fluid and blood," Nat. Rev. Neurol., vol. 9, no. 4, pp. 201-210, 2013.

[17] R. Brouns, B. De Vil, P. Cras, D. De Surgeloose, P. Marien, and P. P. De Deyn, "Neurobiochemical markers of brain damage in cerebrospinal fluid of acute ischemic stroke patients," Clin. Chem., vol. 56, no. 3, pp. 451-458, 2010.

[18] A. J. E. Green et al., "Use of 14-3-3 and other brain-specific proteins in CSF in the diagnosis of variant Creutzfeldt-Jakob disease," J. Neurol. Neurosurg. Psychiatry, vol. 70, no. 6, pp. 744-748, 2001.

[19] A. Leviton and O. Dammann, "Brain damage markers in children.

Neurobiological and clinical aspects," Acta Paediatr., vol. 91, no. 1, pp. 9-13, 2002.

[20] L. G. Tina et al., "S100B protein and near infrared spectroscopy in preterm and term newborns," Front Biosci
(Elite Ed), vol. 2, pp. 159-164, 2010.

[21] E. Trakas et al., "Serum neuronal biomarkers in neonates with congenital heart disease undergoing cardiac surgery," Pediatr. Neurol., vol. 72, pp. 56-61, 2017.

[22] J. M. Simsic, D. S. Atkinson Jr, P. M. Kirshbom, and B. Weissman, "Biomarkers Identify Newborns at Risk following Cardiac Surgery."

[23] R. P. Pironkova et al., "Brain injury with systemic inflammation in newborns with congenital heart disease undergoing heart surgery," Exp. Ther. Med., vol. 14, no. 1, pp. 228-238, 2017.

[24] B. Schmitt et al., "Serum and CSF levels of neuron-specific enolase (NSE) in cardiac surgery with cardiopulmonary bypass: a marker of brain injury?," Brain Dev., vol. 20, no. 7, pp. 536-539, 1998.

[25] V. Chock and H. C. Lee, "Neurodevelopmental outcomes for infants born with congenital heart disease," Neoreviews, vol. 15, no. 8, pp. e344-e353, 2014.

[26] B. S. Marino et al., "Neurodevelopmental outcomes in children with congenital heart disease: evaluation and management: a scientific statement from the American Heart Association," Circulation, vol. 126, no. 9, pp. 1143-1172, 2012.

[27] M. T. Donofrio and C. Limperopoulos, "Impact of congenital heart disease on fetal brain development and injury," Curr. Opin. Pediatr., vol. 23, no. 5, pp. 502-511, 2011.

[28] C. Hernández-García, A. Rodríguez- 
Rodríguez, and J. J. Egea-Guerrero,

"Brain injury biomarkers in the setting of cardiac surgery: Still a world to explore," Brain Inj., vol. 30, no. 1, pp. 10-17, 2016, doi:

10.3109/02699052.2015.1079733.

[29] M. Rundgren, T. Cronberg, H. Friberg, and A. Isaksson, "Serum neuron specific enolase-impact of storage and measuring method," BMC Res. Notes, vol. 7, no. 1, pp. 1-7, 2014.

[30] K. Ishida, T. Gohara, R. Kawata, K. Ohtake, Y. Morimoto, and T. Sakabe, "Are serum S100 $\beta$ proteins and neuronspecific enolase predictors of cerebral damage in cardiovascular surgery?," $J$. Cardiothorac. Vasc. Anesth., vol. 17, no. 1, pp. 4-9, 2003. 